\title{
Delta-doped electron-multiplied CCD with absolute quantum efficiency over $50 \%$ in the near to far ultraviolet range for single photon counting applications
}

\author{
Shouleh Nikzad ${ }^{1, *}$, Michael E. Hoenk ${ }^{1}$, Frank Greer $^{1}$, Blake Jacquot ${ }^{1}$, Steve Monacos ${ }^{1}$, \\ Todd J. Jones ${ }^{1}$, Jordana Blacksberg ${ }^{1}$, Erika Hamden², David Schiminovich², \\ Chris Martin ${ }^{3}$, and Patrick Morrissey ${ }^{3}$ \\ ${ }^{1}$ Jet Propulsion Laboratory, California Institute of Technology, Pasadena, California 91109, USA \\ ${ }^{2}$ Columbia University, New York, New York 10025, USA \\ ${ }^{3}$ California Institute of Technology, Pasadena, California 91125, USA \\ ${ }^{\star}$ Corresponding author: Shouleh.Nikzad@jpl.nasa.gov \\ Received 19 July 2011; accepted 2 September 2011; \\ posted 18 November 2011 (Doc. ID 151268); published 20 January 2012
}

\begin{abstract}
We have used molecular beam epitaxy (MBE) based delta-doping technology to demonstrate nearly $100 \%$ internal quantum efficiency (QE) on silicon electron-multiplied charge-coupled devices (EMCCDs) for single photon counting detection applications. We used atomic layer deposition (ALD) for antireflection (AR) coatings and achieved atomic-scale control over the interfaces and thin film materials parameters. By combining the precision control of $\mathrm{MBE}$ and $\mathrm{ALD}$, we have demonstrated more than $50 \%$ external QE in the far and near ultraviolet in megapixel arrays. We have demonstrated that other important device performance parameters such as dark current are unchanged after these processes. In this paper, we briefly review ultraviolet detection, report on these results, and briefly discuss the techniques and processes employed. (C) 2012 Optical Society of America
\end{abstract}

OCIS codes: $\quad$ 040.0040, 160.0160, 310.0310, 250.0250, 040.7190, 040.6070.

\section{Introduction and Brief Review of UV Detection}

Ultraviolet, optical, and near infrared imaging and spectroscopy instruments have a wide range of applications in space exploration, medical diagnostics and interoperation planning, consumer electronics, and communications. High efficiency ultraviolet (UV) photon detection is essential for many instrument and sensor concepts and has been the focus of recent research endeavors in astrophysics, planetary science, biology, and biomedical fields. For example, future NASA UV/optical missions beyond the Hubble Space Telescope (HST) and the Galaxy Evolution

$1559-128 \mathrm{X} / 12 / 030365-05 \$ 15.00 / 0$

(C) 2012 Optical Society of America
Explorer (GALEX) will require significant detector advances, particularly in quantum efficiency $(\mathrm{QE})$, noise, resolution, and number of pixels, in order to enable major new scientific discoveries.

UV detectors can be classified into two major categories: (1) vacuum tube technologies that combine a UV sensitive photocathode, e.g., CsI or CsTe, with a gain component, and an electron detector; (2) solidstate devices based on silicon or wide bandgap semiconductors. Electron bombarded CCDs (EBCCDs) and microchannel plates (MCPs) are in the first category. Traditionally, for space and laboratory applications, researchers have chosen MCPs because of their low noise and high gain, which enhances the signal to noise ratio at low light levels and enables photon counting; however, these devices have 
relatively low UV QE ( 10\%) and require high voltage power supplies for their operation. Solid-state detectors, on the other hand, offer significant advantage in size, mass, power, reliability, and manufacturability compared to the vacuum tube based technology of MCPs. To surpass the performance of MCPs and enable major new scientific impacts, a solid-state UV detector with stable, high QE, low noise and moderate gain is required. Furthermore, solidstate detector arrays with these capabilities would fulfill the need for instrument compactness and reduction of instrument complexity, both of which are at a premium, especially in instruments designed and built for space and biomedical applications.

Wide bandgap materials, such as gallium nitride $(\mathrm{GaN})$, are intrinsically insensitive to visible photons, and these materials are being used for UV detector arrays as well as UV photocathodes. For UV detector arrays, a multilayer structure of $\mathrm{GaN}$ or its alloys is grown by a technique such as metal organic chemical vapor deposition (MOCVD) or molecular beam epitaxy (MBE) on a sapphire substrate. An array of $\mathrm{p}-\mathrm{i}-\mathrm{n}$ diodes is then fabricated from this material and hybridized to a complementary metal oxide semiconductor (CMOS) readout array. There have also been reports of wide bandgap photodiode arrays with gain [1-3]. These detectors still suffer from higher defect density, higher noise, and poor uniformity in comparison with silicon detectors. On the other hand, an immense investment has been made in order to produce silicon detectors with very low noise, low dark current, and very large imager formats. Significantly, silicon sensors have now become viable for photon counting applications using new architectures to achieve gain, such as electronmultiplied charge-coupled devices (EMCCDs) [4-6] with lateral gain and low noise CMOS sensors with in-pixel avalanche photodiodes $[7,8]$.

Using silicon arrays for UV detection historically has posed a challenge. Because of the shallow absorption length of UV photons into the material, siliconimaging arrays are not suitable in a conventional front-illuminated configuration for UV detection. Instead silicon arrays must be operated in a thinned, back-illuminated configuration, in which photons are incident on the bare silicon surface opposite from the circuitry. While back illumination avoids absorption and scattering in the gate and pixel structures on the front surface, back-illuminated silicon arrays require effective methods of surface passivation to achieve high, stable QE, especially in the UV. Without effective surface passivation, traps at the $\mathrm{Si}_{-} \mathrm{SiO}_{2}$ interface can dynamically interact with photo-generated charge, resulting in poor $\mathrm{QE}$, unstable response (QE hysteresis), and high dark current. Various surface passivation methods have been developed for back-illuminated detectors in order to improve the UV response, reduce surface-generated dark current, and stabilize the overall QE. Ion implantation techniques and chemisorption techniques applied to thinned backside-illuminated charge-coupled de- vices (CCDs) have achieved high quantum efficiencies in the near UV region of the spectrum (above $250 \mathrm{~nm}$ ). Extending these techniques to detect shorter wavelength photons, these detectors encounter instability and low QE.

NASA's Jet Propulsion Laboratory (JPL), California Institute of Technology, has developed a surface passivation technology based on molecular beam epitaxial growth of a silicon delta layer on backilluminated silicon detector arrays. Delta-doping technology uniquely achieves atomic-scale control over the surface bandstructure of a silicon-imaging array, resulting in nearly internal $\mathrm{QE}$ from the extreme ultraviolet (EUV) through near infrared regions of the spectrum. Additionally, these devices have demonstrated very low surface-generated dark current and exhibited no QE hysteresis [9-11].

In this paper, we report the demonstration of a high and stable far UV QE in a solid-state detector array that possesses gain and low noise. This has been achieved by combining three independent technologies. EMCCDs provide the necessary gain and low noise, while delta doping provides near $100 \%$ internal QE, surface passivation, and high near surface electric fields, and atomic layer deposition (ALD) provides high quality antireflection (AR) coatings.

EMCCDs have been invented at $\mathrm{e} 2 \mathrm{v}$ in their low light level L3Vision CCD design [5]. This approach leverages all the advantages of the mature CCD technology while enabling single photon detection by appending a gain register to the end of the serial register prior to the readout amplifier. Electron multiplication in the gain register is achieved by increasing the gate voltages in the second serial clock phase, which causes a small avalanche multiplication at each stage in the register. Using this method, cumulative electron-multiplication gains of more than 1000 can be achieved.

The combination of MBE for delta doping and ALD AR coatings provides atomic-scale control over interface properties. Delta doping passivates the silicon surface to achieve high and stable charge collection efficiency (i.e., internal QE). Because the delta-doped surface is only silicon and its native oxide, further enhancement of $\mathrm{QE}$ using AR coatings is possible without significant constraints based on the starting surface. The challenges of developing AR coatings for this part of the spectrum (i.e., the $100-300 \mathrm{~nm}$ region) include silicon's rapidly changing index of refraction, material limitations for having both low absorption and high index, constraints on deposition methods that offer high quality ultrathin layers without affecting the delta-doped layer or the device front-side collection and readout circuitry $[12,13]$. We have demonstrated that the combination of deltadoping technology and ALD achieves the balance necessary to overcome these challenges.

\section{Experimental}

Commercial EMCCDs, i.e., e2v's CCD97s with L3CCDs design, $1024 \times 512$ pixel format, $16 \mu \mathrm{m} 2$ 
pixel size, and $20 \mu \mathrm{m}$ epilayer, were used in this effort. For our purpose, we thinned to $8 \mu \mathrm{m}$ by removing the $p+$ substrate and approximately $12 \mu \mathrm{m}$ of the epilayer using a multistep process starting with the chemical mechanical polishing (CMP) process followed by chemical thinning to achieve a smooth and highly specular silicon surface. Following the thinning, a series of solvent cleaning steps was used to eliminate residual organic material used in processing steps, such as photoresist and waxes. The devices were then delta doped following processes described in detail elsewhere [9,14]. Briefly, using MBE, a $2.5 \mathrm{~nm}$ layer of single crystal silicon with a high density of boron dopant nominally in a single atomic sheet was grown on the back surface of the device. After delta doping, the devices were used for a variety of AR coating processing experiments. To establish the success of combining the deltadoping process with EMCCDs, the first devices were mounted in packages and wire bonded after delta doping without any further processing. Devices were characterized in a fully calibrated vacuum UV system equipped with a vacuum monochrometer, two sources of deuterium and tungsten allowing characterization from 120-700 nm, a differentially pumped device chamber, and National Institute of Standards and Technology (NIST)-calibrated photodiodes for flux measurements. Details of this characterization system are described elsewhere $[15,16]$.

ALD was used for the deposition of ultrathin layers of hafnium oxide and aluminum oxide. The layers were modeled using TFCalc, and they were deposited on the back surface of thinned delta-doped CCDs. The magnesium fluoride AR coating was deposited using thermal evaporation. Work is now underway to deposit the fluoride film by ALD as well.

\section{Results and Discussion}

We first present the result of a bare delta-doped EMCCD in Fig. 1. The uncoated results provide an experimental control for AR coatings by establishing

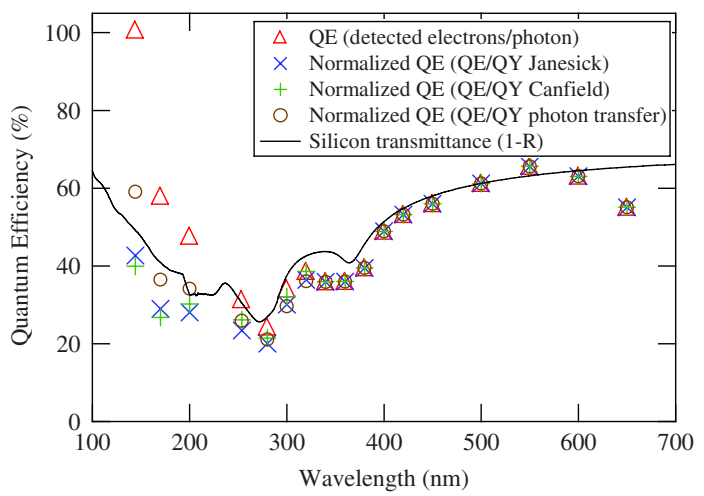

Fig. 1. QE of a $512 \times 1024$ delta-doped EMCCD. The CCD is an e2v L3CCD that was thinned and delta doped at JPL. The solid line is the theoretical reflection limit of the silicon surface. Data is corrected for the multiple electron-hole pair production using two different approximations. Either correction gives a reasonable agreement with transmittance (1-reflection) of silicon. [12] A conservative estimate in the uncertainty of the measurements is $10 \%$. reflection-limited response. Also the bare delta-doped surface response validates the surface passivation. A back-illuminated device should respond at the reflection limit of silicon if there are no other losses for the photo-carriers. If the backside potential well is not eliminated (i.e., if the surface states are not fully passivated), quantum efficiency hysteresis (QEH) will occur. QEH is highly undesirable, especially in space imaging and spectroscopy applications. For example, the HST Wide Field Camera requires a periodic, intense flood illumination to remove this $\mathrm{QEH}$. This has been discussed in detail elsewhere $[17,18]$. Figure 1 shows the QE of a delta-doped EMCCD or delta-doped L3CCD. For comparison, silicon transmittance, or 1- $R$ where $R$ is reflectivity of a bare silicon surface, has also been plotted (see [12] and references therein). It is important to note that in reporting our data, we account and correct for the multiple electron-hole pair, i.e., quantum yield (QY) produced by higher energy UV photons and present true $\mathrm{QE}$ or the detected photons as a percentage of incident photons. At higher wavelengths or lower photon energies, the QY is approximately unity, and at wavelengths shorter than $360 \mathrm{~nm}^{19}$, this number is qualitatively proportional to the incident photon energies. This proportionality has been modeled and measured for higher photon energies including x-rays and has been reported for silicon by different groups as a constant number or as a function of incident photon energy $[15,19-21]$. A detailed discussion can be found elsewhere $[16,20,22]$. Accounting for QY and normalizing to that number is important, because failure to do so results in erroneously high QE. In Fig. 1, we directly demonstrate this phenomenon by presenting our data in three ways: (1) the ratio of measured electrons to the number of incident photons (analyzed signal not normalized to QY); (2) and (3) the ratio of detected photons to the number

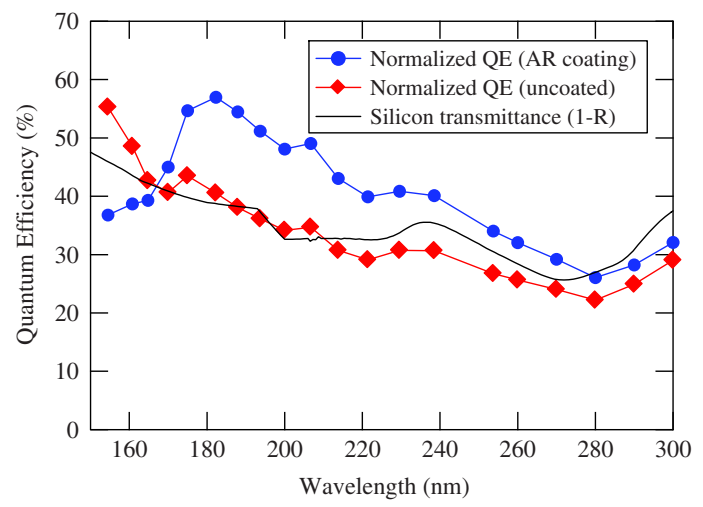

Fig. 2. Far UV and UV response of a delta-doped electron-multiplied L3CCD. The response of the coated region shows significant enhancement in efficiency over the uncoated region in the region targeted by the aluminum oxide AR coating. The uncoated region $\mathrm{QE}$ is in good agreement with the silicon transmittance data within the uncertainties of measurements. [12,16] The QY method used to determine the true $\mathrm{QE}$, (i.e., fraction of photons detected) was direct measurement at each wavelength in our laboratory using the photon transfer technique [15,21]. 


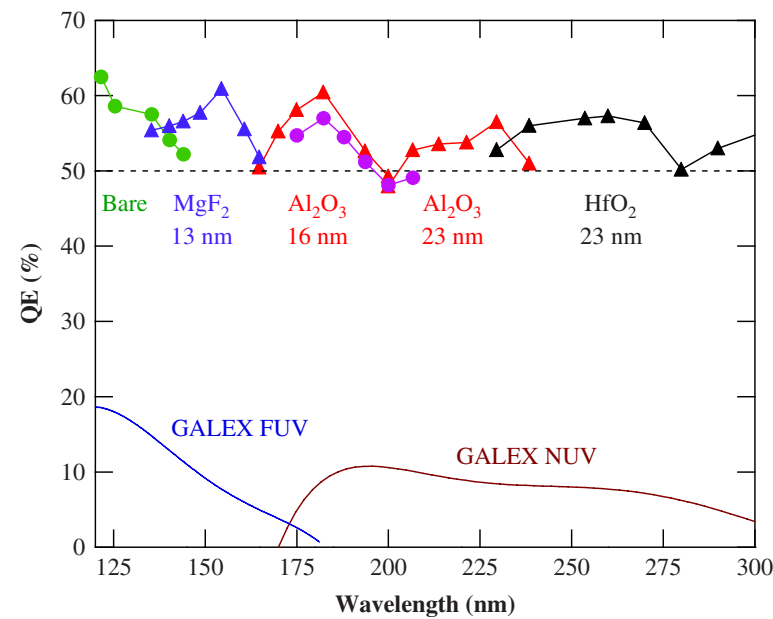

Fig. 3. Response of the AR-coated, delta-doped arrays in the entire 100-300 $\mathrm{nm}$ region. Each region was coated by one simple coating. Further enhancements can be achieved by adding additional complexity in the coatings. In the third band, the response of AR-coated, delta-doped EMCCD $\left(16 \mathrm{~nm} \mathrm{Al}_{2} \mathrm{O}_{3}\right)$ is plotted on the same plot as the conventional CCDs.

of incident photons using two different QY sets of values to correct and normalize for multiple electrons per photon in the measured signal. As shown in Fig. 1, with either correction and within the uncertainties of the measurement, the device responds at reflection limit or nearly $100 \%$ internal QE. Also shown in Fig. 1, the ratio not normalized for $\mathrm{QY}$ will result in an erroneously high QE.

The response of another delta-doped EMCCD array was measured after the device was partially AR coated by shadow masking using a silicon piece to cover a portion of the device. $\mathrm{An}^{\mathrm{Al}_{2} \mathrm{O}_{3} \text { layer was }}$ modeled to cover the $170-200 \mathrm{~nm}$ region and allow enhancement of the response so that the final measured $\mathrm{QE}$ is $>50 \%$ over a significant wavelength range. Figure $\underline{2}$ shows the $\mathrm{QE}$ response of the bare

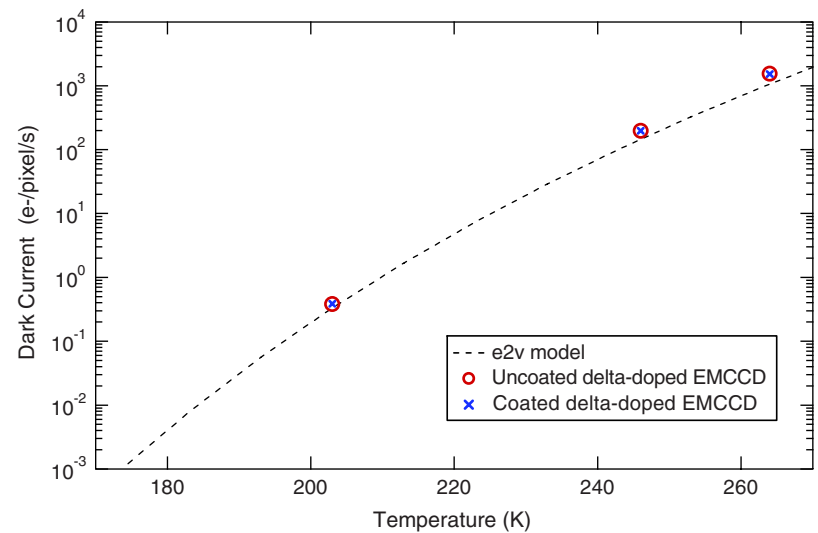

Fig. 4. Dark current measurements for a partially AR-coated, delta-doped electron-multiplied L3CCD. The coated and uncoated data are derived from measurements of different regions of the same device, with the uncoated region defined by a contact shadow mask during the AR coating deposition process. Both sets of data follow the dark current model developed by $\mathrm{e} 2 \mathrm{~V}$ as a function of temperature for their devices [25]. delta-doped region and the AR-coated region of the same device. As shown in Fig. 2, significant enhancement is achieved due to the single $\mathrm{Al}_{2} \mathrm{O}_{3}$ layer film.

Other AR coatings were designed to cover the rest of the 100-300 $\mathrm{nm}$ region of the spectrum and to conserve resources; these were all demonstrated on thinned and delta-doped conventional n-channel CCDs with Cassini mission heritage, which are readily available to our group at JPL. In Fig. $\underline{3}$, the detectors' responses are plotted as a function of incident photon wavelength showing that $\mathrm{QE}>50 \%$ is achieved in the UV. The response of an AR-coated and delta-doped EMCCD is also plotted and within the uncertainties is in agreement with the other device measurement. For comparison, the response of the MCP detector flown on the GALEX mission is also plotted [23,24].

In addition to $\overline{\mathrm{QE}}$ measurements, we characterized the device for dark current, another measure of a well passivated back-illuminated silicon array, by combining three technologies. Figure 4 shows the results of dark current measurement as a function of temperature for both regions of a single device that was partially AR coated in one region. Also, plotted on Fig. 4 is the e2v model of device dark current as a function of temperature presented as a dashed line [25]. It is shown in Fig. 4 that the device behaves according to the model and both the AR-coated and bare regions delta-doped array follow the expected behavior as a function temperature.

\section{Conclusions}

In conclusion, we have demonstrated high $\mathrm{QE}$ in the far UV in solid-state arrays that possess gain and low noise. Features of this deep UV detector technology address the key requirements for UV photon counting detection with solid-state detector arrays. We have achieved nearly $100 \%$ internal QE or reflectionlimited response using MBE delta doping of EMCCDs. Furthermore, we have demonstrated 50\% measured external QE for the 100-300 nm spectral range, by designing and depositing AR coatings using ALD and thermal evaporation. Single photon counting is achieved by the electron-multiplied design, and efficient detection of UV photons is achieved using an ultrathin p-type delta-doped layer to passivate and accumulate the back surface. The significance of these results is enabling solid-state detectors for achieving high QE in far UV in a platform of a low noise device that possesses gain and therefore enables single photon counting. Additionally, unlike photo-emissive devices such as MCPs or EBCCDs, the combination of these techniques provides a powerful detector in the UV and far UV without use of high voltage.

The work presented in this paper was performed by the Jet Propulsion Laboratory (JPL), California Institute of Technology, under a contract with NASA. We gratefully acknowledge the generous collaborative effort by e2v Inc. and helpful discussions with Peter Pool, Paul Jorden, and Paul Jerram of e2v. 


\section{References}

1. R. McClintock, J. L. Pau, C. Bayram, B. Fain, P. Giedraitis, M. Razeghi, and M. P. Ulmer, "III-nitride avalanche photodiodes," Proc. SPIE 7222, 72220U (2009).

2. J. C. Carrano, D. J. H. Lambert, C. J. Eiting, C. J. Collins, T. Li, S. Wang, B. Yang, A. L. Beck, R. D. Dupuis, and J. C. Campbell, "GaN avalanche photodiodes," Appl. Phys. Lett. 76, 924-926 (2000).

3. R. M. Field, J. Larry, J. Cohn, L. Paninski, and K. L. Shepard, "A low-noise, single-photon avalanche diode in standard $0.13 \mu \mathrm{m}$ complementary metal-oxide-semiconductor process," Appl. Phys. Lett. 97, 211111 (2010).

4. J. Hynecek, "Impactron-A new solid state image intensifier," IEEE Trans. Electron Devices 48, 2238-2241 (2001).

5. P. Jerram, P. Pool, R. Bell, D. Burt, S. Browring, S. Spencer, M. Hazelwood, I. Moody, N. Catlett, and P. Heyes, "The LLCCD: low light imaging without the need for an intensifier," Proc. SPIE 4306, 178-186 (2001).

6. C. D. Mackay, R. N. Tubbs, R. Bell, D. Burt, P. Jerram, and I. Moody, "Sub-electron read noise at $\mathrm{MHz}$ pixel rates," Proc. SPIE 4306, 289-298 (2001).

7. C. Niclass, A. Rochas, P. A. Besse, and E. Charbon, "Design and characterization of a CMOS 3-D image sensor based on single photon avalanche diodes," IEEE J. Solid-State Circuits 40, 1847-1854 (2005).

8. N. Faramarzpour, M. J. Deen, S. Shirani, and Q. Fang, "Fully integrated single photon avalanche diode detector in standard CMOS $0.18 \mu \mathrm{m}$ technology," IEEE Trans. Electron Devices 55, 760-767 (2008).

9. M. E. Hoenk, P. J. Grunthaner, F. J. Grunthaner, R. W. Terhune, M. Fattahi, and H. F. Tseng, "Growth of a deltadoped silicon layer by molecular-beam epitaxy on a chargecoupled device for reflection-limited ultraviolet quantum efficiency," Appl. Phys. Lett. 61, 1084-1086 (1992).

10. J. Blacksberg, M. E. Hoenk, S. T. Elliott, S. E. Holland, and S. Nikzad, "Enhanced QE of high purity silicon imagers by ultralow temperature surface modification using Sb-doping," Appl. Phys. Lett. 87, 254101 (2005).

11. S. Nikzad, M. E. Hoenk, P. J. Grunthaner, R. W. Terhune, F. J. Grunthaner, R. Wizenread, M. Fattahi, and H-F. Tseng, "Delta-doped CCDs: high QE with long-term stability at UV and visible wavelengths," Proc. SPIE 2198, 907-915 (1994).

12. E. T. Hamden, F. Greer, M. E. Hoenk, J. Blacksberg, M. R. Dickie, S. Nikzad, C. Martin, and D. Schiminovich, "UV anti-reflection coatings for use in silicon detector design," Appl. Opt. 50, 4180-4188 (2011).

13. F. Greer, E. Hamden, B. C. Jacquot, M. E. Hoenk, J. Blacksberg, S. P. Monacos, M. R. Dickie, D. Schiminovich, and S. Nikzad, manuscript in preparation for Nature Nanotechnology.
14. M. E. Hoenk, T. J. Jones, M. R. Dickie, F. Greer, T. J. Cunningham, E. R. Blazejewski, and S. Nikzad, "Delta-doped back-illuminated CMOS imaging arrays: progress and prospects," Proc. SPIE 7419, 74190T (2009).

15. B. C. Jacquot, S. P. Monacos, T. J. Jones, J. Blacksberg, M. E. Hoenk, and S. Nikzad, "Characterization and absolute QE measurements of delta-doped N-channel and P-channel CCDs," Proc. SPIE 7742, 774201 (2010).

16. B. C. Jacquot, S. P. Monacos, M. E. Hoenk, F. Greer, T. J. Jones, and S. Nikzad, "A system and methodologies for absolute quantum efficiency measurements from the vacuum ultraviolet through the near infrared," Rev. Sci. Instrum. 82, 043102 (2011).

17. S. M. Baggett, J. W. MacKenty, R. A. Kimble, T. Borders, B. Hilbert, S. Deustua, V. Kozhurina-Platais, K. S. Long, A. Riess, R. Gilliland, R. J. Hill, and J. Kalirai, "WFC3 detectors: on-orbit performance," Proc. SPIE 7731, 773138 (2010).

18. N. R. Collins, N. Boehm, G. Delo, R. D. Foltz, R. J. Hill, E. Kan, R. A. Kimble, E. Malumuth, R. Rosenberry, A. Waczynski, Y. Wen, S. Baggett, H. Bushouse, S. Deustua, J. Kim-Quijano, J. MacKenty, A. Martel, and E. Sabbi, "Wide field camera 3 CCD quantum efficiency hysteresis: characterization and mitigation," Proc. SPIE 7439, 74390 (2009).

19. L. R. Canfield, R. E. Vest, R. Korde, H. Schmidtke, and R. Desor, "Absolute silicon photodiodes for $160 \mathrm{~nm}$ to $254 \mathrm{~nm}$ photons," Metrologia 35, 329-334 (1998).

20. J. R. Janesick, Photon Transfer : DN [lambda] (SPIE, 2007).

21. P. F. Morrissey, S. R. McCandliss, and P. D. Feldman, "Vacuumultraviolet quantum efficiency of a thinned, backsideilluminated charge-coupled device," Appl. Opt. 34, 4640-4650 (1995).

22. S. Nikzad, T. J. Cunningham, M. E. Hoenk, R. P. Ruiz, D. M. Soules, and S. E. Holland, "Direct detection of 0.1-20 keV electrons with delta doped, fully depleted, high purity silicon p-i-n diode arrays," Appl. Phys. Lett. 89, 182114-182116 (2006).

23. C. Martin, T. Barlow, T. Conrow, K. Forster, P. G. Friedman, R. McLean, M. Morais, , P.F. Morrissey, F. Raison, D. Schiminovich, T. Small, T. Wyder, and C. K. Xu, "The Galaxy Evolution Explorer," Proc. SPIE 4854, 336-350 (2003).

24. D. C. Martin, M. Seibert, J. D. Neill, D. Schiminovich, K Forster, R. M. Rich, B. Y. Welsh, B. F. Madore, J. M. Wheatley, P. Morrissey, and T. A. Barlow, "A turbulent wake as a tracer of 30,000 years of Mira's mass loss history," Nature 448, 780-783 (2007).

25. e2v_technologies, e2V Low-Light Technical Note 4: Dark Signal and Clock-Induced Charge in L3VisionTM CCD Sensors (2004). 